\title{
Migraine is comorbid with multiple sclerosis and associated with a more symptomatic MS course
}

\author{
Ilya Kister - A. B. Caminero - T. S. Monteith • \\ A. Soliman - T. E. Bacon - J. H. Bacon - J. T. Kalina • \\ M. Inglese $\cdot$ J. Herbert $\cdot$ R. B. Lipton
}

Received: 23 April 2010/Accepted: 21 June 2010/Published online: 13 July 2010

(C) Springer-Verlag 2010

\begin{abstract}
The objectives of this study were: (1) to assess relative frequency of migraine in multiple sclerosis (MS) patients using the validated self-administered diagnostic questionnaire, and to compare the migraine rates in MS outpatients to age- and gender-matched historical population controls; (2) to compare clinical and radiographic characteristics in MS patients with migraine and headachefree MS patients. We conducted a cross-sectional study to assess the demographic profiles, headache features and clinical characteristics of MS patients attending a MS clinic using a questionnaire based on the American Migraine Prevalence and Prevention (AMPP) study. We compared the relative frequency of migraine in MS clinic patients and
\end{abstract}

I. Kister $(\bowtie)$ · A. B. Caminero · A. Soliman .

T. E. Bacon - J. H. Bacon · J. T. Kalina · J. Herbert Department of Neurology, NYU-MS Care Center, NYU School of Medicine, 301 E 17th St, New York, NY 10003, USA e-mail: ilya.kister@gmail.com

A. B. Caminero

Department of Neurology, Hospital Nuestra Señora de Sonsoles, Ávila, Spain

T. S. Monteith

Department of Neurology, NYU School of Medicine,

New York, NY 10003, USA

J. H. Bacon

Department of Psychology, Stern College for Women,

Yeshiva University, New York, NY, USA

\section{Inglese}

Department of Radiology, NYU School of Medicine,

New York, NY, USA

R. B. Lipton

Department of Neurology and the Montefiore Headache Center, Albert Einstein College of Medicine, Bronx, USA
AMPP cohort. We also compared clinical and radiographic features in MS patients with migraine to an MS control group without headache. Among 204 MS patients, the relative frequency of migraine was threefold higher than in population controls both for women [55.7 vs. 17.1\%; prevalence ratio $(\mathrm{PR})=3.26, p<0.001]$ and men $(18.4$ vs. $5.6 \%$; PR $=3.29, p<0.001)$. In a series of logistic regression models that controlled for age, gender, disease duration, $\beta$-interferon use, and depression, migraine in MS patients was significantly associated $(p<0.01)$ with trigeminal and occipital neuralgia, facial pain, Lhermitte's sign, temporomandibular joint pain, non-headache pain and a past history of depression. Migraine status was not significantly associated with disability on patient-derived disability steps scale or T2 lesion burden on brain MRI. Migraine is three-times more common in MS clinic patients than in general population. MS-migraine group was more symptomatic than the MS-no headache group.

Keywords Migraine - Multiple sclerosis ·

Magnetic resonance imaging - Symptomatology ·

Comorbidity $\cdot$ Cross-sectional study

\section{Introduction}

The possibility of a link between migraine and multiple sclerosis (MS) was recognized more than half a century ago when Compston and McAlpine [1] reported that 2\% of MS patients developed migraines within 3 months of their first relapse. The association between migraine and MS was observed in two case-control series $[2,3]$ that predated introduction of the International Classification of Headache Disorders (ICHD-2) [4]. Four of five subsequent controlled studies $[2,3,5-7]$ and several uncontrolled studies that 
used ICHD-2 criteria confirmed that headache prevalence is elevated among persons with MS [8-11]. Among these is a population-based study from Sicily that reported a doubling of headache prevalence in MS patients compared to controls [5]. Only one study did not detect a statistically significant difference in headaches between MS patients and historical controls [7], possibly due to very high prevalence of primary headaches in the local population.

It is not clear if the association between headache and MS is specific to migraine. Pöllmann et al. [10, 11] observed that among interferon-treated MS patients, migraine was predominant headache type while tensiontype headache predominated among those not treated with interferon.

The question of whether co-existing migraine in an MS patient affects the symptomatology, comorbidity, and course of MS has not been well explored. An observed association between presence of migraine and relapsingremitting course of MS [3, 9], has not been reported in all studies. As some comorbidities have recently been shown to be risk factors for increased MS severity [12] this issue acquires special relevance.

The objectives of our study were threefold: (1) to compare the relative frequency of migraine in an MS specialty clinic and in population controls using the validated self-administered diagnostic questionnaire from the American Migraine Prevalence and Prevention (AMPP) study [13]; (2) to compare the 'MS-migraine group' with the 'MS-no headache' group with respect to symptom profile and co-existing diagnoses, with special attention to psychiatric disorders, which are known to be more frequent among migraineurs [14-16]; (3) to compare radiographic (MRI) markers of disease in MS migraineurs and in headache-free MS.

\section{Methods}

NYU-MS Care Center serves an ethnically and socioeconomically diverse population of over 2,400 MS patients in the greater New York area. Eligible patients were over 18 years of age, had diagnosis of MS by the revised McDonald criteria and fluent understanding of written English. They completed a questionnaire at the time of routinely scheduled clinic visits over a 3-month period from October 2006 to January 2007. The study was approved by Institutional Review Board of New York University Medical Center.

We aimed to collect over 200 responses as this would allow us to separate observed prevalence of migraines from the historical controls (13\%) [17], if the observed prevalence is less than $9.4 \%$ or more than $19.5 \%$, with confidence intervals on prevalence estimates of $>95 \%$.
Questions on headache frequency and characteristics were identical to those in the validated diagnostic questionnaire included in the American Migraine Prevalence and Prevention (AMPP) study, the largest epidemiological study of migraine to date. This questionnaire assesses the characteristics of up to three patient-identified headaches and has a sensitivity of $100 \%$ and specificity of $82 \%$ for an ICHD-2-based gold standard diagnosis of migraine [17]. Diagnoses were assigned using identical data and procedures in the NYU-MS Care Center and in the AMPP study [13]. Prevalence of various headaches in MS patients from the NYU-MS Care Center was compared to that in AMPP cohort. Migraine severity was assessed using the validated Migraine Disability Assessment (MIDAS) tool [18]. Comorbidities were assessed in the MS patients using the same instruments as in the AMPPPatient Health Questionnaire (PHQ-9) for depression, Patient Health Questionnaire (PHQ) for anxiety and Allodynia Symptom Check list for allodynia. Additional questions, not included in the AMPP, addressed MS duration and treatment, fatigue (Fatigue Severity Scale), daytime sleepiness (Epworth Sleepiness Scale), and brainstem and visual symptoms using questions developed specifically for this study. We also assessed rates of 'episodic neurologic dysfunction' (END) defined as 'a new neurological symptom, or significant worsening of preexisting symptom(s) of more than $24 \mathrm{~h}$ in duration'. Although END definition mirrors that of MS relapse in the McDonald's criteria, we use the former term because our patients were not necessarily examined at the time of their new or worsening symptoms and so we were unable to corroborate patients' report of worsening with objective findings on examination and exclude possibility of intercurrent infection/stressor. Thus, ENDs could represent bona fide relapses or 'pseudo-exacerbations' (unmasking of previous deficits due to physiological or psychological stressors). The definition of END was provided in the questionnaire, along with a list of typical neurologic symptoms reported during an MS relapse. Patients were asked to estimate the number of ENDs they had experienced over the previous year. Patients' disability level was assessed with Patient-Derived Disability Steps. PDDS is a surrogate measure of the Expanded Disability Status Scale (EDSS) and correlates well with the EDSS in both cross-sectional and longitudinal assessments [19].

Brain MRIs completed on participating patients within 2 years of the survey were reviewed by an MS neurologist and an expert in MS neuroradiology blind to the patients' clinical history. MRI scans included T2-weighted, and preand post-contrast T1-weighted sequences. All scans were done at 1.5-T magnet machine with exception of five scans that were done at 3-T magnet and two scans at 0.6-T magnet. The number of hyperintense lesions on brain 
T2-weighted scans, hypointense lesions on T1-weighted scans and gadolinium-enhancing lesions on post-contrast T1-weighted sequence were recorded, as were the number of T2 hyperintense lesions in periventricular white matter, subcortical white matter, corpus callosum, brainstem and basal ganglia. Lesions involving a given structure were considered as separate, and therefore counted individually, if regions of normal signal intensity were present between slices.

Group comparisons were carried out using $t$ tests for continuous variables and Chi-squared test for differences in proportions. A series of logistic regression models were used to assess the association of migraine on a variety of outcome measures in persons with MS. The main predictor variable was migraine status. We controlled for age, gender, disease duration, $\beta$-interferon use and depression (coded as 'yes'/'no' variable, with 'yes' defined as score of 10 or more on PHQ-9, which corresponds to moderate depression cut-off). Separately modeled dichotomous outcome variables included common MS and pain symptoms: optic neuritis, trigeminal neuralgia, facial pain, occipital neuralgia, Lhermitte's sign, cognitive, visual and brainstem symptoms, restless legs syndrome, painful spasms, body pain, allodynia, temporomandibular joint syndrome and analgesic use.

We also used linear regression to assess the impact of age, gender, disease duration, beta-interferon use and depression on continuously distributed outcome variables. To assess the influence of migraine disability on symptom profiles, we conducted analyses stratified by MIDAS grade using the four-level disability scoring grading [Grade I, none-mild (0-5); Grade II, moderate (6-10); Grade III, severe (11-20); Grade IV (>20)] [20]. Statistical analyses were carried out using SPSS Statistics v. 17.0 software.

\section{Results}

Frequency and characteristics of migraines in MS patients and AMPP historical controls

The questionnaire was distributed to 225 patients in the waiting room of NYU-MS Center, and 204 (90\%) returned a completed questionnaire; $64 \%$ of patients (131) reported "a headache within the past year not related to trauma, infection or medication". Of the 131 patients with a headache, $94(72 \%)$ met ICHD-2 criteria for migraines and the remaining $37(28 \%)$ for tension-type headaches. There were ten migraineurs $(9.4 \%)$ who had a second headache type that met criteria for tension-type headache. The patients with migraine and non-migraine headache were included in the migraine group. In this report, we compare MS migraineurs (MS-Mig)—this group consisted of 94 patients-with headache-free MS (MS-NH) patients, 73 patients. In general, patients with tension-type headache and MS had clinical characteristics intermediate between those of the non-headache controls and the migraine cases. Data on tension-type headache group will be presented in a separate publication.

AMPP population consisted of 162,576 responders, of whom 18,968 individuals met ICHD-2 criteria for migraine yielding an unadjusted 1-year period prevalence estimate of $11.7 \%$ (5.6\% for men and $17.1 \%$ for women) [13]. Relative frequency of migraine in MS outpatients was more than threefold higher than in the AMPP population controls both for men (18.4 vs. $5.6 \%, \mathrm{RR}=3.29$, $p<0.001)$ and for women (55.7 vs. 17.1, $\mathrm{RR}=3.36$, $p<0.001)$. Migraine frequency among women with MS was significantly higher for each age groups (20-29, 30-39, 40-49 and 50 and above) compared to AMPP controls. There were too few men with both MS and migraine to allow for age-adjusted analysis.

Thirty-six percent of MS-Mig group had auras. Among those with aura, most were visual (44.1\% of auras), or visual/sensory (38.2\%), and a minority, sensory only $(17.6 \%)$. The frequency of migraine with aura and distribution of aura types is nearly identical to that of migraineurs in the AMPP cohort [20]. Migraine disability, as assessed with MIDAS, did not differ in the MS and in the AMPP samples: $77 \%$ of MS-Mig and $75 \%$ of the AMPP migraineurs had mild-to-moderate migraine-related disability (MIDAS Grades 1 and 2), while the remainder fell into migraine Grades 3 and 4. Six patients in the MS cohort (6.4\%) had chronic migraines; all six met criteria for medication overuse headache.

Demographic characteristics, past medical and family history

The demographic characteristics, frequencies of co-existing conditions and family history of MS and migraines in MS-Mig and MS-NH are presented in Table 1. The two groups were similar with respect to age (47 \pm 13 vs. $43 \pm 11$ years), and disease duration (13 \pm 11 vs. $12 \pm 11$ years), but MS-Mig had a larger fraction of women compared to MS-NH (90 vs. 57\%, $p<0.001$ ).

MS-Mig were more likely than MS-NH to report history of depression (36 vs. $21 \%, p<0.028$ ), and scored higher on PHQ-9 scales for depression (8.2 vs. 4.7, $p<0.001)$. More MS migraineurs reported a history of mononucleosis compared to headache-free MS patients (20 vs. 5\%, $p<0.01$ ), while the rates of other co-existing conditions were similar.

Family history of MS in first-degree relatives was not different in the two groups, but family history of migraines was 2.5 times higher (49\%) among MS-Mig than in 
Table 1 Demographic characteristics, co-existing medical conditions and family history in MS-Mig and MS-NH groups

\begin{tabular}{llll}
\hline & MS-NH & MS-Mig & $p$ \\
\hline$N$ & 73 & 94 & - \\
Age in years (mean $\pm \mathrm{SD}$ ) & $47 \pm 13$ & $43 \pm 11$ & $\mathrm{NS}$ \\
$\%$ Female* & $57 \%$ & $90 \%$ & $<0.001$ \\
Disease duration in years (mean $\pm \mathrm{SD})$ & $13 \pm 11$ & $12 \pm 11$ & $\mathrm{NS}$ \\
\% Interferon beta-1 use & $71 \%$ & $64 \%$ & $\mathrm{NS}$ \\
Co-existing conditions & & & \\
Hypertension & $12 \%$ & $15 \%$ & $\mathrm{NS}$ \\
Diabetes & $0 \%$ & $4 \%$ & $\mathrm{NS}$ \\
Seizures & $1 \%$ & $4 \%$ & $\mathrm{NS}$ \\
Syncope & $1 \%$ & $7 \%$ & $\mathrm{NS}$ \\
Thyroid disease & $8 \%$ & $10 \%$ & $\mathrm{NS}$ \\
Fibromyalgia & $0 \%$ & $4 \%$ & $\mathrm{NS}$ \\
Mononucleosis* & $5 \%$ & $20 \%$ & 0.006 \\
Autoimmune disease & $4 \%$ & $12 \%$ & $\mathrm{NS}$ \\
Bipolar disorder & $4 \%$ & $3 \%$ & $\mathrm{NS}$ \\
Anxiety spectrum disorder & $8 \%$ & $16 \%$ & $\mathrm{NS}$ \\
Depression & $21 \%$ & $36 \%$ & 0.028 \\
Current smoker & $18 \%$ & $19 \%$ & $\mathrm{NS}$ \\
Family history & & & \\
Family history of MS & $17 \%$ & $10 \%$ & $\mathrm{NS}$ \\
Family history of migraine* & $19 \%$ & $49 \%$ & $<0.001$ \\
\hline
\end{tabular}

MS-Mig MS and migraine group, MS-NH MS-no headache group, $N S$ non-significant

$* p<0.01$

MS-NH (19\%), and 3.5 times higher in the MS-Mig with aura subset (59\%) compared to MS-NH.

Episodic neurologic dysfunction in MS migraineurs and headache-free MS patients

Episodic neurologic dysfunction (END) that lasted more than $24 \mathrm{~h}$ was reported more frequently by MS-Mig group compared to MS-NH group. In the MS-Mig group, 28\% had three or more ENDs over past year, and only $20 \%$ had none. By contrast, only $10 \%$ of MS-NH reported three or more ENDs and 32\% had none. The fraction of patients with either one or two ENDs was similar in the two groups. Patients who had never experienced an END likely had primary progressive form of MS; these patients constituted a relatively larger proportion of the MS-NH (9\%) than of the MS-Mig (3\%) group. The trend towards the increased number of ENDs was most pronounced in the subset of patient with migraine with aura $(N=29)$. In the MS-Mig with aura subset, $52 \%$ reported three or more ENDs over past year and only 7\%, no ENDs.
Pain-related and non-pain-related symptom profiles

The prevalence of specific pain-related symptoms, such as Lhermitte's sign, occipital and trigeminal neuralgia, facial pain, temporomandibular joint pain, spasms, and restless legs syndrome, was 2-5 times higher in MS-Mig than in MS-NH (Table 2). Frequent, non-headache-related allodynia was reported by $26 \%$ of migraineurs and only $4 \%$ of headache-free patients. Over $20 \%$ of the MS-Mig group required analgesics for non-headache reasons on a daily basis, compared to $6 \%$ in MS-NH group (Table 2). Over-the-counter medication, NSAIDS and acetaminophen, were used for headache by 80 of the 94 migraineurs $(85 \%)$.

MS-Mig had considerably more cognitive, psychiatric, brainstem and visual symptoms, and scored higher on depression and anxiety scales (PHQ-9, PHQ), fatigue severity scale and Epworth sleepiness scale than MS-NH patients (Table 3).

To control for potential confounders, we modeled the relationship between migraine status and MS related outcomes using logistic regression to account for age, gender, disease duration, $\beta$-interferon use and depression (defined as score of 10 or more on PHQ-9). MS related outcomes associated with migraine at the $p<0.01$ level include trigeminal neuralgia, occipital neuralgia, facial pain, painful spasms, Lhermitte's sign, temporomandibular joint pain and non-headache pain. Migraine was not a predictor of cognitive, brainstem or visual symptoms, restless legs syndrome, allodynia or daily pain medication use. Depression was a significant predictor of visual and cognitive problems. Gender was a predictor of painful spasms and disease duration was a predictor of Lhermitte's sign and brainstem symptoms. The results of logistic regression analyses are presented in Tables 4 and 5 as odds ratios with 95\% confidence.

We modeled the relationship of migraine status to a series of continuous outcomes using linear regression. These models were adjusted for the same independent variables used in the logistic models (migraine status, age, gender, disease duration, $\beta$-interferon use and depression). Migraine was a significant predictor of anxiety and nearly reached significance for Epworth sleepiness scale $(p=0.013)$, while depression and disease duration were significant predictors of fatigue. Age and gender were not predictive of any outcome variable.

Importantly, despite the more symptomatic course, the disability of MS migraineurs, as assessed by PatientDerived Disability Score (PDDS) was mild-nearly all could ambulate without assistive device—and was similar to those without headache. 
Table 2 Painful symptoms and analgesic use in MS-Mig and MS-NH groups

$* p<0.01$

Table 3 Non-pain symptoms in MS-Mig and MS-NH groups

$* p<0.01$

${ }^{\text {a }}$ Double vision, facial weakness, deafness, abnormal taste, vertigo

\begin{tabular}{lclrrr}
\hline & MS-NH (\%) & MS-Mig (\%) & $p$ & OR & CI \\
\hline Brief, shooting pains into jaw* & 5 & 27 & $<0.001$ & 9.7 & $2.1-44$ \\
Shooting pains into back of head/neck* & 11 & 45 & $<0.001$ & 9.2 & $2.7-32$ \\
Facial pain* & 10 & 32 & 0.001 & 6.6 & $1.6-27$ \\
Temporomandibular joint syndrome* & 7 & 30 & $<0.001$ & 4.8 & $1.4-16$ \\
Lhermitte's sign* & 18 & 48 & $<0.001$ & 5.2 & $1.9-14$ \\
Painful spasms* & 29 & 48 & 0.012 & 3.7 & $1.4-9.6$ \\
Restless legs feeling, movement-relieved* & 33 & 57 & 0.002 & 2.1 & $0.87-4.8$ \\
Frequent allodynia (not during headache)* & 4 & 26 & $<0.001$ & 5.3 & $1.2-23$ \\
Daily use of pain medication for non-headache* & 6 & 21 & 0.006 & 7.3 & $1.2-46$ \\
\hline
\end{tabular}

\begin{tabular}{llllll}
\hline & MS-NH & MS-Mig & $p$ & OR & CI \\
\hline Optic neuritis* & $49 \%$ & $77 \%$ & $<0.001$ & 2.8 & $1.2-6.4$ \\
Visual problems interfere with reading & $22 \%$ & $32 \%$ & NS & 0.64 & $0.25-1.7$ \\
Brainstem symptoms*, & $27 \%$ & $53 \%$ & 0.001 & 2.4 & $1.0-5.7$ \\
Cognition "definitely affected" & $24 \%$ & $38 \%$ & 0.039 & 1.8 & $0.67-4.9$ \\
Depression score (PHQ-9) & 4.7 & 8.2 & $<0.001$ & NA & NA \\
Anxiety score (PHQ) & 4.1 & 6.5 & $<0.001$ & NA & NA \\
Fatigue severity score & 3.6 & 5.0 & $<0.001$ & NA & NA \\
Hours of sleep per night & 6.7 & 6.8 & NS & NA & NA \\
Epworth sleep scale score* & 5.6 & 8.1 & $<0.001$ & NA & NA \\
\hline
\end{tabular}

Table 4 Predictors of non-pain MS symptoms, according to a multivariate logistic regression model using a forward selection criteria

\begin{tabular}{llrl}
\hline & Optic neuritis & Visual problems & Cognitive symptoms \\
\hline No migraine versus migraine & $0.300(0.145-0.621)$ & & $0.197(0.084-0.458)$ \\
No depression versus depression & & $0.232(0.103-0.522)$ & $3.178(1.427-7.078)$ \\
No IFN $\beta$ use versus IFN $\beta$ use & & &
\end{tabular}

Values are OR and 95\% CI after forward selection criterion, $p<0.01$

Age, gender, disease duration, IFN $\beta$ usage, migraine and depression where not significant predictors of brainstem symptoms; age, gender and disease duration were not significant predictors of any MS symptoms; $p \leq 0.01$

Side-effects of interferon-beta 1

Interferon-beta (IFN $\beta$-1) use was similar in the two groups (71\% for MS-NH and 64\% for MS-Mig, $p>0.1$ ), and the remainder were using glatiramer acetate or were on no medication. MS-Mig reported more side-effects to interferons than MS-NH: 32\% of MS-Mig patients had flu-like symptoms following 'all or almost all injections' compared to only $13 \%$ headache-free patients. Furthermore, $34 \%$ of migraineurs on IFN $\beta$ - $1 \mathrm{a}$ had a headache within $24 \mathrm{~h}$ of the injection "always or almost always", while the no-headache group did not have post-injection headache.

\section{Radiological characteristics of MS-Mig and MS-NH groups}

Brain MRIs performed within 2 years of the survey were available for 138 out of 204 patients in the study (67\%), including 65 MRIs of MS-Mig and 49 of MS-NH patients. The remaining MRIs were of patients with tension-type headache, and will be discussed in a separate publication. Table 6 compares the radiographic findings of the two groups. There were no differences in the number or distribution of $\mathrm{T} 2$ hyperintense lesions or the number of gadolinium-enhancing lesions in the MS-Mig and MS-NH groups. There was a trend for more $\mathrm{T} 1$ hypointense lesions in the headache-free group $(p<0.05)$.

\section{Discussion}

The overall prevalence of headache in our MS sample was $64 \%$, which is within the range reported in several previous studies [8-11]. Majority of headache seen in MS patients, $72 \%$, were migraines. In comparison with AMPP historical controls, migraine was threefold more common in MS 


\begin{tabular}{|c|c|c|c|c|c|c|}
\hline \multirow{4}{*}{ 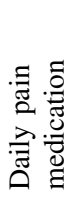 } & \multirow{3}{*}{ 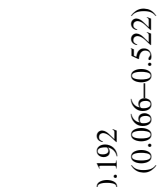 } & \multirow{3}{*}{ 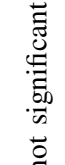 } & \multicolumn{4}{|c|}{ Table 6 Brain MRI characteristics of MS-Mig and MS-NH groups } \\
\hline & & & & MS-NH & MS-Mig & $p$ \\
\hline & & & No. of brain MRI & 49 & 65 & \\
\hline & & $=$ & \# of $\mathrm{T} 2$ hyperintense lesions & 21.5 & 19.6 & NS \\
\hline & & $\lesssim$ & \# of $\mathrm{T} 2$ hyperintense lesions $<5 \mathrm{~mm}$ & 7.0 & 8.2 & NS \\
\hline & & $\mathscr{D}_{0}^{\circ}$ & $\mathrm{T} 2$ hyperintense lesions by location & & & \\
\hline 当 & & $=$ & Periventricular & 7.3 & 5.8 & \\
\hline$\stackrel{8}{0}$ & & 罙 & Subcortical & 9.9 & 11.3 & \\
\hline$\varangle$ & 0 & Z & Basal ganglia & 1.1 & 0.3 & \\
\hline & f & $=$ & Corpus callosum & 2.0 & 1.3 & \\
\hline & m & 急 & Brainstem & 0.6 & 0.4 & \\
\hline & $\frac{5}{7}$ & $\Xi$ & \# of T1 gadolinium-enhancing lesions & 0.3 & 0.5 & NS \\
\hline ป气 & $\because \ddot{0}$ & $\ddot{g}$ & \# of T1 hypointense lesions & 6.0 & 3.3 & $<0.05$ \\
\hline
\end{tabular}

outpatients. The increased relative frequency of migraine is consistent with most controlled studies of headache in MS $[2,3,6]$.

Several possible reasons for the association can be suggested. One possibility is that headaches are directly attributable to MS ('secondary headaches'). One consideration that mitigates against this possibility is that MSMig group had an elevated family history of migraine in comparison to those without migraine. If the excess of migraine-like headaches were attributable to MS, this result would not be expected. Furthermore, migraine characteristics in the MS patient and general population such as the distribution of migraine-related disability grades (MIDAS grade), and the ratio of migraine with aura to migraine without aura were nearly identical in the MS clinic and the general population $[13,20]$. If 'MS migraine' were a distinct entity, it would be more probable that its characteristics would be distinguishable from those of primary migraines. Accurate data on temporal relationship between MS and migraine could help clarify the nature of association. To this end, longitudinal studies in which mechanistic hypotheses will be developed and explored should be pursued.

Our study is the first to investigate both clinical and radiographic profiles of MS in patients with and without migraine. The MS-Mig group had a remarkable increase in the frequency of both pain-related and non-pain-related symptoms. MS-Mig group also reported significantly more episodes of neurologic dysfunction and were likely to have progressive form of MS, confirming a previously observed association between migraines and the relapsing form of MS [9].

We carried out separate analyses comparing headachefree patients to the subset of MS-Mig with very mild migraines (MIDAS Grade 1) and more severe migraines (Grades 2-4). We observed significant increases in ENDs rates and symptom frequencies in both mild migraine and 
severe migraine subgroup compared to non-headache group (data not shown). This threshold effect suggests that the presence of migraine, rather than attack frequency and severity, accounts for the increased symptom frequency in MS-Mig subset.

Why do MS migraineurs appear to be so much more prone to experience new or worsening neurological symptoms than MS patients who do not have migraines? It seems unlikely that ENDs represent a direct expression of migraine aura since most auras subside within an hour and we specifically enquired about symptoms whose duration exceeds $24 \mathrm{~h}$. Furthermore, increases in symptom frequency were observed both in MS migraineurs with auras and those without. Two potential explanations for increased symptom rate in MS migraineurs may be considered: (1) migraine may trigger more severe inflammation in the brain, in which case, increased number of $\mathrm{T} 2$ hyperintense lesions, and, especially, of $\mathrm{T} 1$ gadoliniumenhancing lesions, would be expected on brain MRIs of MS-Mig compared to MS-NH patients; or, (2) migraineurs may report more symptoms of MS at an equivalent level of brain pathology, inasmuch as they experience altered pain perception and have a decreased pain threshold, a phenomenon thought to be due to central sensitization [21-25]. In the latter case, lesion burden on MRI would not be expected to differ in the two groups.

In our study, T2 and gadolinium-enhancing lesion burden was the same in the two groups, in agreement with a previous report, in which total number of T2 lesions in MS migraineurs did not differ from MS non-migraineurs [26]. Since elevated symptom and END rates in MS-Mig did not translate into greater lesion burden on their MRI, it appears more plausible that the more elaborate symptomatology in the migraineurs is not due to more active MS disease activity in that patient subset, but to their greater susceptibility to manifesting neurologic symptoms. This explanation of increased symptom rates in migraineurs may also account for our finding that patient's disability did not differ between the two groups.

Another hypothesis that is relevant to a subset of migraineurs with aura is that cortical spreading depression of migraine aura causes unmasking of MS symptoms. If this were the case, then we would find that migraineurs with aura (MA) experience even more ENDs than migraineurs without aura. Indeed, $52 \%$ of MA patients reported three or more ENDs over the past year, compared to $38 \%$ in the allmigraineur group, and $10 \%$ in the no-headache group. By contrast, only $7 \%$ of MA patients had no ENDs over the past year, as compared to $20 \%$ of the all-migraineur group and $32 \%$ in the no-headache group (data not shown). Thus, it is possible that migraine aura may trigger prolonged neurological deficits with recovery analogous to the unmasking of deficits in experimental lesions by cortical spreading depression [27]. One practical ramification of this observation is that an MS patient who has migraine with aura may need to be aggressively treated for migraine in order to prevent not only his migraines, but also prolonged episodes of neurological disability that may be triggered by migraine aura.

We conclude that the greater susceptibility of migraineurs to experience symptoms relating to apperception may be due to decreased pain and visual discomfort thresholds in these patients and their proclivity to allodynia [24,28] or to induction of 'pseudoexacerbation' via cortical spreading depression. Whether the discrepancy in symptom frequency between and MS migraineurs and non-migraineurs extends to the non-sensory realms as well (e.g. motor, or autonomic function deficits, or extraocular movement abnormalities) is an important question that cannot be answered with our data.

Migraine is comorbid with depression in the general population [14-16] and our study extends this association to MS population. MS migraineurs reported history of depression at significantly higher rates than headache-free MS patients, and also scored higher on PHQ-9 scale for depression. It is important to note that higher END rates and symptom frequencies in the MS-Mig group cannot be attributed to higher rates of depression in that group as migraine was a significant predictor of most pain-related symptoms even in the logistic regression model that included depression as one of the dependent variables.

Consistent with most previous reports $[6,11,30]$, MS migraineurs tended to be intolerant of IFN $\beta$-1: one in three experienced a headache 'always or almost always' following the injection. Even those with no change in migraine pattern experienced flu-like symptoms at a significantly higher rate than headache-free patients. In clinical practice, it would seem prudent to question all MS migraineurs who are initiated on $\beta$-interferon therapy for any change in headache pattern and for flu-like symptoms since these side-effects may have a considerable detrimental impact on their quality of life and adherence to therapy.

One drawback of our study is that it was conducted in a waiting room of a specialty MS clinic. Although, NYU-MS Center serves patients from all socio-economic strata, and the demographics of our patient population are reflective of MS patients in our area (National MS Society survey, 2007, data on file), clinic patients may be skewed toward the more severe end of the disability spectrum. Moreover, studies carried out within medical institutions tend to overestimate rates of association with comorbid disorders, a phenomenon known as 'Berkson's bias' [29].

Another potential objection to the study is that the elevated frequency of migraines seen in our patient population is due, at least in part, to the wide-spread IFNb-1 use at our 
Center, which could trigger and exacerbate migraines [6, $11,30]$. To address this objection, we conducted logistic regression modeling that was adjusted for interferon use. Migraine remained a significant predictor of most MS symptoms in this analysis as well (Tables 4,5 ).

\section{Conclusions}

Our study demonstrates that relative frequency of migraine is increased threefold in MS patients attending a specialty clinic compared to historical population controls. Migraine disability was considerable among MS patients: nearly one in four was classified in moderate or severe MIDAS grades. Migraines in MS are very common, often disabling, yet potentially treatable. Headache history should therefore be elicited from all MS patients.

A novel result of our study is a description of 'MS migraineur' who may be distinguished from a headachefree counterpart by a higher probability of episodic neurologic dysfunction, pain-related symptoms, and higher rates of depression and anxiety. Population-based, prospective studies with objective documentation of relapses, cognitive and physical disability, regular MRI brain examination and controlled for IFNb-1 use, psychiatric comorbidities, presence of alexithymic trait, are necessary to clarify the nature of the association between migraine and MS, and to confirm our finding that MS migraineurs experience a markedly more symptomatic, but not necessarily more disabling, MS course.

Acknowledgments This study was supported by a Pilot Award PP1471 from the National Multiple Sclerosis Society to Drs. Kister, Herbert, Bacon and Lipton.

Conflict of interest Dr. Kister received funding for research activities from EMD Serono. Dr. Lipton receives research support from the NIH [PO1 AG03949 (Program Director), PO1AG027734 (Project Leader), RO1AG025119 (Investigator), K23AG030857 (Mentor), K23NS05140901A1 (Mentor), and K23NS47256 (Mentor)], the National Headache Foundation, and the Migraine Research Fund; serves on the editorial boards of Neurology and Cephalalgia and as senior advisor to Headache, holds stock options in Neuralieve Inc. and Minster Inc; serves as consultant for Advanced Bionics, Allergan, Inc., Boehringer-Ingelheim, Endo, Glaxo Smith Kline, Kowa, Minster, Merck, Neuralieve and Pfizer and received honoraria from Allergan, Inc, Glaxo Smith Kline and Merck, Inc. Dr. Herbert has consulting agreements with Biogen, TEVA, EMD Serono and Bayer. Dr. Inglese received consulting fees from TEVA Pharmaceuticals. Other authors have nothing to disclose.

\section{References}

1. Compston N, McAlpine D (1952) Some aspects of the natural history of disseminated sclerosis. Q J Med 21:135-167
2. Watkins SM, Espir M (1969) Migraine and multiple sclerosis. J Neurol Neurosurg Psychiatry 32:35-37

3. Rolak LA, Brown S (1990) Headaches and multiple sclerosis: a clinical study and review of the literature. J Neurol 237:300-302

4. Headache Classification Committee of the International Headache Society (2004) Classification and diagnostic criteria for headache disorders, cranial neuralgias and facial pain, 2nd edn. Cephalalgia 24(Suppl 1):1-116

5. Nicoletti A, Patti F, Lo Fermo S, Liberto A, Castiglione A, Laisa P, Garifoli A et al (2008) Headache and multiple sclerosis: a population-based case-control study in Catania, Sicily. Cephalalgia 28:1163-1169

6. Vacca G, Marano E, Brescia Morra V, Lanzillo R, De Vito M, Parente E, Orefice G (2007) Multiple sclerosis and headache comorbidity. A case-control study. Neurol Sci 28:133-135

7. Putzki N, Pfriem A, Limmroth V, Yaldizli O, Tettenborn B, Diener HC, Katsarava Z (2009) Prevalence of migraine, tensiontype headache and trigeminal neuralgia in multiple sclerosis. Eur J Neurol 16:262-267

8. D'Amico D, La Mantia L, Rigamonti A, Usai S, Mascoli N, Milanese C, Bussone G (2004) Prevalence of primary headaches in people with multiple sclerosis. Cephalalgia 24:980-984

9. Villani V, Prosperini L, Ciuffoli A, Pizzolato R, Salvetti M, Pozzilli C, Sette G (2008) Primary headache and multiple sclerosis: preliminary results of a prospective study. Neurol Sci 29(Suppl 1):S146-S148

10. Pöllmann W, Erasmus LP, Feneberg W, Straube A (2006) The effect of glatiramer acetate treatment on pre-existing headaches in patients with MS. Neurology 66:275-277

11. Pöllmann W, Erasmus LP, Feneberg W, Then Bergh F, Straube A (2002) Interferon beta but not glatiramer acetate therapy aggravates headaches in MS. Neurology 59:636-639

12. Marrie RA, Horwitz R, Cutter G, Tyry T, Campagnolo D, Vollmer T (2009) Comorbidity delays diagnosis and increases disability at diagnosis in MS. Neurology 72:117-124

13. Lipton RB, Bigal ME, Diamond M, Freitag F, Reed ML, Stewart WF et al (2007) Migraine prevalence, disease burden, and the need for preventive therapy. Neurology 68:343-349

14. Swartz KL, Pratt LA, Armenian HK, Lee LC, Eaton WW (2000) Mental disorders and the incidence of migraine headaches in a community sample: results from the Baltimore Epidemiologic Catchment area follow-up study. Arch Gen Psychiatry 57:945950

15. Merikangas KR, Angst J, Isler H (1990) Migraine and psychopathology. Results of the Zurich cohort study of young adults. Arch Gen Psychiatry 47:849-853

16. Breslau N, Lipton RB, Stewart WF, Schultz LR, Welch KM (2003) Comorbidity of migraine and depression: investigating potential etiology and prognosis. Neurology 60:1308-1312

17. Lipton RB, Scher AI, Kolodner K, Liberman J, Steiner TJ, Stewart WF (2002) Migraine in the United States: epidemiology and patterns of health care use. Neurology 58:885-894

18. Stewart WF, Lipton RB, Whyte J, Dowson A, Kolodner K, Liberman JN, Sawyer J (1999) An international study to assess reliability of the Migraine Disability Assessment (MIDAS) score. Neurology 53:988-994

19. Hohol MJ, Orav EJ, Weiner HL (1995) Disease steps in multiple sclerosis: a simple approach to evaluate disease progression. Neurology 45:251-255

20. Diamond S, Bigal ME, Silberstein S, Loder E, Reed M, Lipton RB (2007) Patterns of diagnosis and acute and preventive treatment for migraine in the United States: results from the American Migraine Prevalence and Prevention study. Headache 47:355363

21. Aurora SK, Wilkinson F (2007) The brain is hyperexcitable in migraine. Cephalalgia 27:1442-1453 
22. Burstein R, Yarnitsky D, Goor-Aryeh I, Ransil BJ, Bajwa ZH (2000) An association between migraine and cutaneous allodynia. Ann Neurol 47:614-624

23. Kitaj MB, Klink M (2005) Pain thresholds in daily transformed migraine versus episodic migraine headache patients. Headache 45:992-998

24. Mathew NT, Kailasam J, Seifert T (2004) Clinical recognition of allodynia in migraine. Neurology 63:848-852

25. Scher AI, Stewart WF, Lipton RB (2006) The comorbidity of headache with other pain syndromes. Headache 46:1416-1423

26. Gee JR, Chang J, Dublin AB, Vijayan N (2005) The association of brainstem lesions with migraine-like headache: an imaging study of multiple sclerosis. Headache 45:670-677
27. Tietelbaum P, Cytawa J (1965) Spreading depression and recovery from lateral hypothalamic damage. Science 147:61-63

28. Bigal ME, Ashina S, Burstein R, Reed ML, Buse D, Serrano D, Lipton RB et al (2008) Prevalence and characteristics of allodynia in headache sufferers: a population study. Neurology 70:1525-1533

29. Berkson J (1946) Limitation to application of four-fold table analysis to hospital data. Biom Bull 2:47-53

30. La Mantia L, D’Amico D, Rigamonti A, Mascoli N, Bussone G, Milanese C (2006) Interferon treatment may trigger primary headaches in multiple sclerosis patients. Mult Scler 12:476-480 